\title{
A avaliação de serviços em Audiologia: concepções e perspectivas
}

\section{Evaluation of hearing health services: concepts and perspectives}

\author{
Maria Cecília Bevilacqua ${ }^{1}$, Tatiana Mendes de Melo², Marina Morettin ${ }^{3}$, Andréa Cintra Lopes ${ }^{4}$
}

\begin{abstract}
RESUMO
A avaliação dos serviços vem ocupando posição de destaque no planejamento e gestão em saúde, uma vez que um sistema efetivo pode reordenar a execução das ações e serviços para contemplar as necessidades da população alvo e otimizar os recursos financeiros. Embora existam algumas experiências internacionais na avaliação dos serviços de saúde auditiva, sua contribuição é limitada, devido às particularidades da Política de Atenção à Saúde Auditiva de cada país. No Brasil, o atendimento integral ao deficiente auditivo foi garantido pela Portaria GM/MS n 2 2.073/04, com a proposta da organização de uma rede hierarquizada, regionalizada e integrada na Atenção básica, Média e Alta Complexidade. O aprimoramento desta Política é uma consequência natural, depois do início de sua implantação, a fim de constituir serviços públicos de saúde auditiva com qualidade, não somente no ato de sua criação, mas também e principalmente, na manutenção e no aprimoramento constante do processo de atendimento de pessoas com deficiência auditiva, assegurando a efetividade do processo. Para tanto, faz-se imprescindível a formação profissional e o incentivo de discussões referentes à avaliação da qualidade dos serviços de saúde auditiva do Sistema Único de Saúde.
\end{abstract}

Descritores: Avaliação de serviços de saúde; Indicadores de qualidade em assistência à saúde; Sistema único de saúde; Audiologia; Perda auditiva

\section{INTRODUÇÃO}

De acordo com as estimativas realizadas pela Organização Mundial da Saúde (OMS), 278 milhões de indivíduos no mundo têm perda auditiva moderada à profunda bilateral $\mathrm{e}$ $80 \%$ destes vivem em países em desenvolvimento ou subdesenvolvidos. A estimativa é que sejam necessários 32 milhões de aparelhos de amplificação sonora individual (AASI) a cada ano nos países em desenvolvimento e apenas 750.000 são distribuídos ${ }^{(1)}$.

No Brasil, esta estimativa é muito difícil de ser avaliada, devido à carência de pesquisas acerca da prevalência da deficiência auditiva na população, em âmbito nacional. Existem apenas dois trabalhos com base populacional que oferecem

Trabalho realizado no Departamento de Fonoaudiologia da Faculdade de Odontologia de Bauru da Universidade de São Paulo - USP - Bauru (SP), Brasil.

(1) Professora Titular do Departamento de Fonoaudiologia da Faculdade de Odontologia de Bauru da Universidade de São Paulo - USP - Bauru (SP), Brasil.

(2) Mestre, Professora do Curso de Fonoaudiologia da Universidade de Guarulhos - UNG - Guarulhos (SP), Brasil.

(3) Pós-graduanda (Doutorado) em Epidemiologia pela Faculdade de Saúde Pública da Universidade de São Paulo - USP - São Paulo (SP), Brasil.

(4) Doutora, Professora do Departamento de Fonoaudiologia da Faculdade de Odontologia de Bauru da Universidade de São Paulo - USP - Bauru (SP), Brasil.

Endereço para correspondência: Tatiana Mendes de Melo. Centro de Pesquisas Audiológicas. R. Silvio Marchione, 3-20, Caixa Postal 1501, Bauru (SP), Brasil, CEP: 17012-900. E-mail: cpa@centrinho.usp.br

Recebido em: 20/1/2009; Aceito em: 20/5/2009 informações a respeito das perdas auditivas incapacitantes ${ }^{(2-3)}$. De acordo com esses estudos, calcula-se prevalência de 4,8\% a 6,8\% (para o Norte e Sul do país, respectivamente) de deficiências auditivas incapacitantes e que necessitam de AASI ou implante coclear.

Baseando-se nessas informações pode-se afirmar que, para um país com dimensões continentais como o Brasil, a demanda de adaptação de AASI seria ao redor de 18 milhões de pessoas ${ }^{(4)}$. Esta demanda está muito longe de ser atendida, tanto pela distribuição dos serviços de saúde auditiva no território nacional, quanto pelo número de AASI produzidos. Esta é uma realidade que se repete ao redor do mundo e agrava-se principalmente nos países em desenvolvimento.

Diante da estimativa do número de deficientes auditivos em nível internacional e nacional, faz-se necessária a otimização dos recursos financeiros empregados na intervenção audiológica $^{(5)}$, a fim de possibilitar melhor benefício para esta população. Para tanto, torna-se imprescindível a identificação de fatores que contribuem para a qualidade do atendimento audiológico prestado ${ }^{(6)}$.

Na Audiologia, têm sido estudados o benefício e satisfação do usuário em relação ao uso do AASI como forma de avaliar os resultados da intervenção. Porém, muitas vezes, este tipo de análise não é suficiente para a avaliação da qualidade dos serviços de saúde auditiva. A avaliação e o uso de indicadores de qualidade nestes serviços é uma temática pouco difundida no cenário internacional e nacional.

Neste sentido, o presente estudo tem o intuito de abordar alguns conceitos teóricos acerca da avaliação e indicadores 
de qualidade dos serviços de saúde, bem como contextualizar o atual panorama desta temática frente aos serviços de saúde auditiva do Sistema Único de Saúde (SUS). Para tanto, o levantamento bibliográfico foi conduzido com os descritores avaliação de serviços de saúde, indicadores de qualidade em assistência à saúde e Sistema Único de Saúde, em base eletrônica de dados de acesso público, tais como: LILACS, Medline e SciELO; sem restrição no ano de publicação. Como o foco do presente estudo é a avaliação de serviços no âmbito do SUS, foram priorizados trabalhos desenvolvidos em nível nacional.

\section{REVISÃO DE LITERATURA}

\section{Avaliação da qualidade dos serviços de saúde: concep- ções gerais}

A avaliação das ações em saúde vem se destacando entre as iniciativas de planejamento e gestão de práticas deste setor ${ }^{(7)}$, a fim de fornecer informações relevantes para o processo de tomada de decisão, baseado em evidências ${ }^{(8)}$ e pode enfocar a avaliação de programas, de serviços ou de tecnologias. Um sistema de avaliação efetivo deve reordenar a execução das ações e serviços, redimensionando-os de forma a contemplar as necessidades de seu público, dando maior racionalidade ao uso dos recursos financeiros.

A avaliação de programas em saúde tem como foco de análise os programas, ou seja, práticas com macro objetivo voltadas a populações específicas, como por exemplo, a avaliação de programas de triagem auditiva neonatal universal. $\mathrm{Na}$ avaliação de tecnologias em saúde, estudam-se novos recursos tecnológicos (equipamentos e/ou medicamentos). Por sua vez, na avaliação de serviços, o principal aspecto a ser considerado é a qualidade do serviço/atendimento prestado ${ }^{(9)}$.

Nos países desenvolvidos, a partir da década de 50, foi reconhecida a necessidade da realização de estudos que tivessem como objeto a avaliação da qualidade dos serviços de saúde $^{(9-10)}$. Esta necessidade surgiu a partir da expansão dos serviços de saúde, de caráter privado, no qual a qualidade do serviço seria um atributo importante na sua valorização do mercado. No setor público, a qualidade do serviço ganhou destaque como parte dos direitos da população ${ }^{(9)}$.

A incorporação da avaliação como prática sistemática nos serviços de saúde é um recurso válido não só para os usuários e profissionais que atuam no serviço ${ }^{(11)}$, como também para os gestores, que terão informações úteis e concretas para definição de prioridades e, conseqüentemente, reorientação das práticas, além do monitoramento dos programas ${ }^{(12)}$.

De maneira geral, a avaliação da qualidade dos serviços de saúde pode abordar três seguimentos: a estrutura, o processo e os resultados ${ }^{(13)}$, como demonstra a Figura 1. A avaliação da estrutura corresponde às condições físicas, equipamentos, materiais e profissionais presentes no serviço de saúde. É difícil quantificar a influência ou contribuição exata desse componente na qualidade final da assistência prestada, mas é possível falar em termos de tendências - estrutura mais adequada aumenta a probabilidade de a assistência prestada ser de melhor qualidade ${ }^{(13)}$. É recomendado que estes três seguimen- tos sejam avaliados para que haja uma análise correspondente à realidade do serviço avaliado.

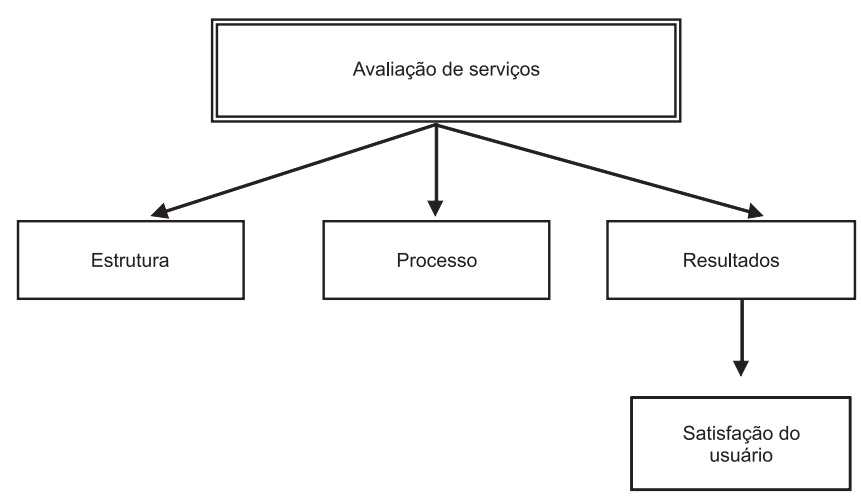

Figura 1. Fluxograma para exemplificar os seguimentos da avaliação da qualidade dos serviços de saúde

A avaliação do processo corresponde ao conjunto de atividades desenvolvidas na relação profissional e paciente (seja na área do diagnóstico ou na reabilitação); a avaliação dos resultados refere-se às mudanças verificadas na condição de saúde do paciente, bem como a satisfação do usuário decorrente do cuidado prestado ${ }^{(13)}$.

Um dos modelos mais conhecidos de avaliação da qualidade dos serviços é a Acreditação, utilizado essencialmente em nível hospitalar, no qual o estabelecimento deve atender a padrões de qualidade previamente estabelecidos em cada área de trabalho, agrupados em três níveis: nível 1 - Segurança (estrutura), nível 2 - Organização (processo), nível 3 - Práticas de Gestão e Qualidade (resultados). Para ser acreditado, o hospital é avaliado por uma organização independente (Instituição Acreditadora) e a coleta de dados dá-se pela observação no local, verificação de prontuários e contatos com os profissionais do serviço.

Independente do pressuposto teórico envolvido na avaliação de serviços de saúde, a construção de indicadores destaca-se como importante ferramenta na análise da qualidade dos serviços de saúde e deve mensurar a qualidade nos três seguimentos: estrutura - processo - resultados ${ }^{(8,14)}$.

Ao construir um indicador, primeiramente é necessário escolher o referencial teórico sobre o qual ele será fundamentado. Em seguida, o objeto estudado deve ser claramente definido para que o indicador possa quantificar os domínios que compõe o objeto de estudo ${ }^{(8)}$. Para cada realidade é preciso examinar os indicadores mais apropriados para atender às necessidades de determinado serviço e os especialistas deverão participar da seleção de indicadores em cada seguimento ${ }^{(15)}$.

No momento da construção do indicador é necessário considerar as características fundamentais de um indicador ideal, a saber: validade - o indicador deve ter alta sensibilidade e alta especificidade; confiabilidade - o indicador deve reproduzir os mesmos resultados quando aplicado em condições similares; disponibilidade - os dados básicos para o cálculo do indicador devem estar disponíveis ou serem fáceis de conseguir; simplicidade - deve ser fácil de analisar e interpretar pelos usuários da informação; relevância - deve responder a prioridades de saúde na tomada de decisão pelos gestores do serviço; custo- 
efetividade - os resultados obtidos pela tomada de decisões baseada nestes indicadores devem justificar o investimento de tempo e recursos para a construção dos mesmos ${ }^{(16)}$.

Um dos principais objetivos do uso de indicadores é melhorar a gestão e a qualidade da assistência oferecida. O acompanhamento do resultado desses instrumentos pode revelar a eficiência da gestão que está sendo desenvolvida. Eles constituem os sensores de como os serviços estão funcionando, acompanhando e orientando todas as ações e podem detectar as possíveis distorções ou dificuldades que um serviço venha a ter ${ }^{(8)}$.

Neste tipo de análise deve-se considerar não apenas o uso dos indicadores de qualidade, mas também a fonte utilizada para analisar o indicador, isto é, quem irá avaliar o serviço ${ }^{(13)}$ e quem utilizará esses resultados ${ }^{(8,17)}$. De maneira geral, há três grupos que podem contribuir com informações relevantes na avaliação de serviços de saúde e/ou utilizar os indicadores: os profissionais que trabalham no serviço; os gestores e os pacientes usuários do serviço ${ }^{(15)}$.

A avaliação do processo é o indicador mais direto para análise da qualidade da assistência prestada, enquanto que os resultados são indicadores de qualidade, de forma indireta, dos aspectos relacionados à estrutura e ao processo ${ }^{(18)}$. Contudo, a melhor forma para avaliar a qualidade dos serviços de saúde requer um conjunto de indicadores representativos dos três seguimentos da avaliação ${ }^{(12)}$.

\section{Perspectivas na avaliação dos serviços de saúde auditiva}

No Brasil, o atendimento ao deficiente auditivo foi incorporado pelo SUS, no início da década de 90, com a Portaria MS/SAS n 126 de 17 de setembro de 1993, que versa sobre o Implante Coclear. Desde então, houve a publicação de mais algumas Portarias referentes ao Implante, tais como: a MS/ $\mathrm{SAS} \mathrm{n}^{\circ} 211$ de 12 de novembro de 1996 e a MS/GM n ${ }^{\circ} 1278$ de 21 de outubro de 1999 que, embora fossem de extrema importância para o acesso da população ao tratamento, as mesmas não englobavam todos os procedimentos que visam boas práticas de atenção e cuidado à saúde auditiva.

A primeira Portaria referente à regulamentação, no âmbito ambulatorial, do diagnóstico, da adaptação e do acompanhamento de indivíduos deficientes auditivos foi a SAS/MS $\mathrm{n}^{\circ} 432$, publicada em 14 de novembro de 2000. Nos anos subseqüentes à publicação desta Portaria, verificou-se um desequilíbrio entre o número de procedimentos referentes ao diagnóstico e concessão de AASI, em comparação com a produção ambulatorial do procedimento de reabilitação auditiva, havendo assim uma concentração de recursos financeiros em determinadas áreas de atenção à saúde auditiva ${ }^{(19-20)}$. O indivíduo deficiente auditivo não recebia atendimento integral conforme os princípios doutrinários do SUS, uma vez que procedimentos como a terapia fonoaudiológica e as ações de saúde auditiva na atenção básica eram realizadas pontualmente.

Em 2004, o Ministério da Saúde, após sentir a necessidade de rever no país a efetividade dos procedimentos de concessão de AASI, instituiu a Política Nacional de Atenção à Saúde Auditiva por meio da Portaria GM/MS nº 2.073 de 28/09/2004.
Esta Política permitiu um grande avanço no aprimoramento das ações de saúde auditiva do SUS, na medida em que propôs a organização de uma rede hierarquizada, regionalizada e integrada na atenção básica, na Média e Alta Complexidade. Desta maneira, a reabilitação auditiva foi garantida como um todo, contemplando não só o diagnóstico e adaptação do AASI, mas também a promoção e a proteção, bem como terapia fonoaudiológica tanto de adultos quanto de crianças ${ }^{(21)}$.

Tendo uma política estabelecida, evidências são necessárias para mostrar quais padrões de práticas são sadias e efetivas, a fim de justificar a alocação dos recursos financeiros ${ }^{(22)}$. Pacientes com alterações auditivas entram nos serviços de saúde auditiva com o objetivo de melhorar sua função auditiva e, conseqüentemente, o seu desempenho comunicativo. Quando o tratamento é fornecido, como a concessão do AASI ou o serviço de reabilitação, ou os dois, a efetividade do tratamento precisa ser avaliada ${ }^{(23)}$.

Assim, a avaliação de serviços de saúde auditiva deve ser voltada para assegurar a efetividade do tratamento do indivíduo deficiente auditivo, como também otimizar a verba pública. Isto é de vital importância, potencialmente nas áreas que envolvem custo financeiro elevado, como no caso do processo de atendimento das pessoas com deficiência auditiva, no qual o valor dos equipamentos para a identificação e o diagnóstico das deficiências auditivas e dos dispositivos eletrônicos aplicados na reabilitação desta deficiência, requerem estudos que favoreçam a viabilização de serviços desta natureza.

Pesquisadores $^{(24)}$ apresentaram uma proposta de avaliação da qualidade de centros de saúde auditiva infantil na Inglaterra, por meio de questionário aplicado nos serviços do país. Embora algumas instituições estivessem atuando em consonância com a Política de Atenção à Saúde preconizada no país, os resultados obtidos revelaram grande variabilidade na qualidade dos serviços e, conseqüentemente, na intervenção audiológica concedida às crianças deficientes auditivas e suas famílias.

A proposta de avaliação da qualidade também tem sido discutida internacionalmente para a Triagem Auditiva Neonatal (TAN), com a elaboração de instrumentos com base nas respostas dos pais ${ }^{(25-26)}$. De maneira geral, os instrumentos são entregues às famílias para o preenchimento em casa e estas, por sua vez, retornam os questionários para os profissionais. Resultados preliminares apontam alto índice de satisfação das famílias em relação ao serviço. Todavia, a taxa de retorno dos questionários ao Programa não ultrapassou 48\%. Do mesmo modo, a não devolução dos questionários deveria ser investigada, uma vez que este achado também pode ser um indicativo da satisfação/ insatisfação dos usuários destes serviços.

Recentemente, o Hearing and Communication Group liderou a construção de indicadores de qualidade dos serviços de Audiologia da Escócia. Estes indicadores foram elaborados a partir das seguintes dimensões: acesso ao serviço, informação ao paciente, planejamento individual, implantação do planejamento individual, resultados da intervenção, competência profissional e suporte do serviço. A avaliação dos serviços de saúde auditiva está sendo conduzida, por meio destes indicadores, mas os resultados preliminares ainda não foram disponibilizados $^{(27)}$. 


\section{DISCUSSÃO}

A Política de Atenção à Saúde Auditiva não apresenta, até o momento, nenhuma ferramenta padronizada de avaliação, na perspectiva fonoaudiológica, que possa contribuir para a assistência oferecida ao indivíduo deficiente auditivo. Atualmente, a avaliação da qualidade dos serviços de saúde auditiva está pautada na infra-estrutura (instalações físicas, número de profissionais, equipamentos) e no quantitativo da produtividade de procedimentos e adaptações de AASI realizados mensalmente, o que não garante a qualidade dos serviços de saúde auditiva.

A portaria SAS 587 do Ministério da Saúde, em seu Anexo III, apresenta um formulário para vistoria do gestor para fins de credenciamento dos serviços de Audiologia na Média e Alta Complexidade ${ }^{(28)}$. Este formulário, no entanto, aborda um enfoque apenas estrutural que pode ser suficiente em um primeiro momento, isto é, na habilitação dos centros, mas pode levar a conclusões errôneas quando utilizado como único instrumento para acreditação dos serviços já existentes. Os auditores dos serviços de saúde auditiva têm usado instrumentos de avaliação de outras áreas para avaliar esses centros, ficando a dúvida de sua adequação e especificidade para a área da Audiologia.

A contribuição de experiências internacionais na avaliação da qualidade dos serviços de saúde auditiva é limitada, devido às diferenças destes e demais especificidades de cada país. Desta maneira, faz-se imprescindível o desenvolvimento de um grupo de indicadores de acordo com as necessidades dos serviços de saúde auditiva em âmbito nacional. Tais indicadores devem enfocar os três pilares da avaliação da qualidade dos serviços de saúde, isto é, estrutura, processos e resultados; para que, quando analisados conjuntamente, produzam avaliações coerentes para a gestão da saúde auditiva e sinalizem ao fonoaudiólogo e pesquisadores os estudos futuros que devem ser desenvolvidos para o melhor atendimento da população brasileira, com qualidade e baixo custo operacional. Além disto, é necessário socializar informações sobre as experiências já existentes neste campo de atuação ${ }^{(25-27)}$, bem como aprofundar a formação do fonoaudiólogo na área de avaliação dos serviços de saúde.

Um aspecto importante na construção dos indicadores de qualidade dos serviços de saúde auditiva é a fonte utilizada para analisar o indicador, ou seja, quem avaliará o serviço. De maneira geral, são os gestores, os profissionais e os pacientes que utilizam os serviços que podem contribuir com informações relevantes sobre a qualidade do serviço. Como as necessidades e exigências de cada grupo são distintas, os indicadores para cada setor também devem ser diferenciados.

Em 2006, o Conselho Nacional de Desenvolvimento Científico e Tecnológico (CNPq) aprovou a concessão de auxílio financeiro para o desenvolvimento do projeto multicêntrico "Indicadores e proposta de avaliação da qualidade dos serviços de audiologia do Sistema Único de Saúde" (processo número 409613/2006-1), que conta com a participação de serviços de saúde auditiva das regiões Sul, Sudeste, Nordeste e Centrooeste do país.
Este projeto é um dos primeiros esforços no contexto nacional na área de avaliação dos serviços de saúde auditiva e tem como objetivo estudar os indicadores de qualidade dos resultados da intervenção. Dados preliminares apontam que os pacientes que utilizam o SUS geralmente têm um perfil de gratidão aos serviços prestados e não se percebe como um cidadão com direitos perante o serviço público, o que dificulta a análise de indicadores de qualidade sob a perspectiva do usuário.

O nível sócio-cultural destes pacientes é outra dificuldade assinalada, pois muitas vezes, estes indivíduos não conseguem responder a questionários de satisfação sem o auxílio de um profissional do serviço, acarretando em respostas nem sempre independentes da influência do próprio atendimento. Supõe-se que, face à insatisfação, o usuário tem direito de apresentar reclamação. Este é um aspecto interessante, teoricamente bem estruturado, mas difícil de ocorrer na prática, pois, da mesma forma que as pessoas nem sempre estão em condições de exercer seus direitos, nem sempre conseguem erguer a sua voz para sugerir modificações ou reclamar ${ }^{(29)}$. Os serviços apresentam a possibilidade de ouvidoria, mas este setor é ainda pouco utilizado no sentido de ser um local de expressão da voz do usuário.

Outra prerrogativa importante apontada pelos dados preliminares deste estudo é que, para análise da avaliação e construção de indicadores de qualidade dos serviços de saúde auditiva, é necessário levar em consideração as diferenças intrínsecas dos serviços de saúde auditiva do país, não só pelo aspecto geográfico do Brasil como também pela população assistida. Desta forma, é necessária a definição de um nível básico de qualidade para um bom atendimento ao paciente e a otimização da verba pública aplicada nestes serviços para todas as regiões do país.

\section{COMENTÁRIOS FINAIS}

A partir da implantação da Política de Atenção à Saúde Auditiva, em 2004, muitos avanços foram conquistados com o intuito de beneficiar a população deficiente auditiva. A avaliação dos serviços de saúde auditiva vem como uma forma de acrescentar melhorias a esta Política, a partir da otimização da verba pública para o atendimento de qualidade para um maior número de indivíduos com perda auditiva.

Como exposto anteriormente, a avaliação da qualidade dos serviços de saúde deve abordar os seguimentos da estrutura, do processo e dos resultados da intervenção para que haja uma análise real do serviço avaliado. Uma avaliação inicial da estrutura e os resultados da intervenção já estão sendo investigados pelo Anexo III da Portaria SAS 587 e pelo estudo do CNPq, respectivamente, mas ainda falta mobilização na investigação do seguimento do processo.

Muita informação se faz necessária sobre a temática exposta e estudos são de fundamental importância para o estabelecimento de diretrizes para avaliação e construção de indicadores de qualidade para os serviços de saúde auditiva do SUS.

O momento atual é intrigante e novos desafios são lançados a fim de aprimorar o atendimento do indivíduo deficiente auditivo no Brasil. 


\section{AGRADECIMENTOS}

Ao Conselho Nacional de Desenvolvimento Científico e Tecnológico (CNPq) pela concessão do auxílio financeiro para o desenvolvimento do projeto multicêntrico "Indicadores e proposta de avaliação da qualidade dos serviços de Audiologia do Sistema Único de Saúde” (processo número 409613/2006-1).

\begin{abstract}
The evaluation of services has played an important role in health planning and management, since an effective system may reorganize the accomplishment of actions and services to meet the needs of the target population and to optimize financial resources. Although there have been some international experiences in the assessment of hearing health services, their contribution is limited, due to the particularities of each country's Hearing Health Attention Policies. In Brazil, integral assistance to the hearing impaired has been guaranteed by Portaria GM/MS number 2.073/04, with the purpose to organize a hierarchical and regionalized network, integrated in Primary-, Medium- and High-complexity Health Care Attention. The improvement of this Policy is a natural consequence, following its implantation, in order to constitute quality hearing health public services, not only upon its creation, but also and mainly in the constant maintenance and improvement of the process of assisting people with hearing impairment, assuring its effectiveness. Therefore, professional qualification and incentive of discussions regarding the evaluation of the quality of hearing health services provided by Brazil's Unified Health System are indispensable.
\end{abstract}

Keywords: Health services evaluation; Quality indicator, health care; Single health system; Audiology; Hearing loss

\section{REFERÊNCIAS}

1. Mathers C, Smith A, Concha M. Global burden of hearing loss in the year 2000. [Internet]. [cited 2007 Out 10]. Available from: http://www. who.int/entity/healthinfo/statistics/bod_hearingloss.pdf; 2001.

2. Béria JU, Raymann BC, Gigante LP, Figueiredo AC, Jotz GP, Roithmann $\mathrm{R}$, et al. Hearing impairment and socioeconomic factors: a populationbased survey of an urban locality in southern Brazil. Rev Panam Salud Publica. 2007;21(6):381-7.

3. Bevilacqua MC. Levantamento das alterações auditivas da população urbana de Monte Negro - Rondônia/Brasil. Bauru (SP): Universidade de São Paulo, Faculdade de Odontologia de Bauru; 2007. Relatório final: Processo CNPq n479793/2004-2.

4. Bevilacqua MC. Atingir a população na capilaridade do sistema. Rev Audio Infos. 2008;13:17.

5. Gatehouse S. The impact of measurement goals on the design specification for outcome measures. Ear Hear. 2000;21(4 Suppl):100S$105 \mathrm{~S}$.

6. Kohli R, Tan JK, Piontek FA, Ziege DE, Groot H. Integrating cost information with health management support system: an enhanced methodology to assess health care quality drivers. Top Health Inf Manage. 1999;20(1):80-95.

7. Bosi MLM, Uchimura KY. Avaliação da qualidade ou avaliação qualitativa do cuidado em saúde? Rev Saúde Publica $=\mathrm{J}$ Public Health 2007;41(1):150-3.

8. Soárez PC, Padovan JL, Ciconelli RM. Indicadores de saúde no Brasil: um processo em construção. Rev Adm Saúde. 2005;7(27):57-64

9. Novaes HMD. Avaliação de programas, serviços e tecnologias em saúde: revisão. Rev Saúde Publica = J Public Health. 2000;34(5):547-59.

10. Novaes HMD. Pesquisa em, sobre e para os serviços de saúde: panorama internacional e questões para a pesquisa em saúde no Brasil. Cad Saúde Pública = Rep Public Health. 2004;20(Supl 2):S147-73.

11. Martinez MN, Gonçalves OR. Qualidade na atenção à saúde. Brasília Méd. 2000;37(3/4):109-13.

12. Silva LMV, Formigli VLA. Avaliação em saúde: limites e perspectivas. Cad Saúde Pública = Rep Public Health. 1994;10(1):80-91.

13. Donabedian A. The definition of quality and approaches to its assessment. Ann Arbor, Mich.: Health Administration Press; c1980. (Série: His Exploration in quality assessment).
14. Bittar OJNV. Indicadores de qualidade e quantidade em saúde. Rev Adm Saúde. 2001;3(12):21-8.

15. Malik AM, Schiesari LMC. Qualidade na gestão local de serviços e ações de saúde. São Paulo: Faculdade de Saúde Pública da Universidade de São Paulo; 1998. (Série Saúde \& Cidadania- v.3)

16. Klück M, Guimarães JR, Ferreira J, Prompt CA. A gestão da qualidade assistencial do Hospital de Clínicas de Porto Alegre: implementação e validação de indicadores. Rev Adm Saúde. 2002;4(16):27-32

17. Santos IN, Victora CG. Serviços de saúde: epidemiologia, pesquisa e avaliação. Cad Saúde Pública = Rep Public Health. 2004;20(Supl 2):S337-41

18. Donabedian A. The role of outcomes in quality assessment and assurance. QRB Qual Rev Bull. 1992;18(11):356-60. Comment in: QRB Qual Rev Bull. 1993;19(3):78.

19. Miranda GMD, Queiroga BAM, Lessa FJD, Leal MC, Caldas Neto SS. Diagnóstico da deficiência auditiva em Pernambuco: oferta de serviços de média complexidade - 2003. Rev Bras Otorrinolaringol. 2006;72(5):581-6.

20. Carvalho JSA. Satisfação de idosos com aparelhos auditivos concedidos no estado de Tocantins. Arq Int Otorrinolaringol. 2007;11(4):416-26.

21. Brasil. Ministério da Saúde. Gabinete do Ministro. Portaria No 2.073/ GM de 28 de setembro de 2004. Institui a Política Nacional de Atenção à Saúde Auditiva. Diário Oficial da República Federativa do Brasil. 2004.

22. Saunders GH, Chisolm TH, Abrams HB. Measuring hearing aid outcomes - not as easy as it seems. J Rehabil Res Dev. 2005;42(4 Suppl 2):157-68

23. Cook JA, Hawkins DB. Outcome measurement for patients receiving hearing aid services. Laryngoscope. 2007;117(4):610-3.

24. Bamford J, Battersby C, Beresford D, Davis A, Gregory S, Hind S, et al. Assessing service quality in paediatric audiology and early deaf education. Br J Audiol. 2001;35(6):329-38.

25. Mazlan R, Hickson L, Driscoll C. Measuring parent satisfaction with a neonatal hearing screening program. J Am Acad Audiol. 2006;17(4):253-64.

26. MacNeil JR, Liu CL, Stone S, Farrell J. Evaluating families' satisfaction with early hearing detection and intervention services in Massachusetts. Am J Audiol. 2007;16(1):29-56. 
27. The Scottish Government. Draft Quality Standards for Adult Hearing Rehabilitation [Internet]. [cited 2008 Sep 16]. Available from: http:// www.scotlandd.gov.uk/publications/2008/01/09093543/0; 2008.

28. Brasil. Ministério da Saúde. Secretaria de Assistência a Saúde. Portaria $\mathrm{N}^{\circ} 587$ de 07 de outubro de 2004. Determina que as Secretarias de Estado da Saúde dos estados adotem as providências necessárias à organização e implantação das Redes Estaduais de Atenção à Saúde Auditiva e da outras providências. Diário Oficial da República Federativa do Brasil. p. 105, 11 de outubro, 2004.

29. Nunes L. Usuários dos Serviços de Saúde e os seus direitos. Rev Bras Bioética. 2006;2(2):201-19. 